This item was submitted to Loughborough's Research Repository by the author.

Items in Figshare are protected by copyright, with all rights reserved, unless otherwise indicated.

\title{
Solvothermal nanoYAG synthesis: mechanism and particle growth kinetics
}

PLEASE CITE THE PUBLISHED VERSION

http://dx.doi.org/10.1016/j.supflu.2015.09.031

PUBLISHER

(c) Elsevier

VERSION

AM (Accepted Manuscript)

\section{PUBLISHER STATEMENT}

This work is made available according to the conditions of the Creative Commons Attribution-NonCommercialNoDerivatives 4.0 International (CC BY-NC-ND 4.0) licence. Full details of this licence are available at: https://creativecommons.org/licenses/by-nc-nd/4.0/

\section{LICENCE}

CC BY-NC-ND 4.0

\section{REPOSITORY RECORD}

Ramanujam, Prabhu, Vaidhy Vaidhyanathan, J.G.P. Binner, Shaghayegh Ghanizadeh, and Chris Spacie. 2015. "Solvothermal Nanoyag Synthesis: Mechanism and Particle Growth Kinetics". figshare. https://hdl.handle.net/2134/19849. 


\section{Solvothermal nanoYAG synthesis: Mechanism and particle growth kinetics}

${ }^{a, b}$ Ramanujam $P,{ }^{a}$ Vaidhyanathan $B,{ }^{a, b}$ Binner JGP, ${ }^{a, c}$ Ghanizadeh S, and ${ }^{d, e}$ Spacie C, ${ }^{a}$ Department of Materials, Loughborough University, Loughborough, UK.

${ }^{b}$ School of Metallurgy and Materials, University of Birmingham, Birmingham, UK. ${ }^{\mathrm{C}}$ Wolfson School of Mechanical and Manufacturing Engineering, Loughborough University, Loughborough, UK.

${ }^{\mathrm{d} M o r g a n}$ Advanced Materials, Swansea, UK

${ }^{\mathrm{e}}$ Haydale Graphene Industries plc, Swansea, UK

\section{Introduction}

Polycrystalline yttrium aluminium garnet (YAG) has potential for a range of structural and functional applications as a result of its excellent physical, chemical and mechanical properties [1]. For example, with a suitable dopant on the yttrium site in the crystal structure, excellent optical properties can be achieved for laser and white LED applications $[2,3]$. The ceramic is also being researched for other uses such as a radar dome [4] and as a highly creep-resistant material at high temperatures [5]. With a cubic crystal structure, YAG does not suffer from birefringence when transparent; the latter may be achieved by either having a very large grain size, which leads to poor strength, or a very fine grain size that is smaller than the wavelength of visible light. In both cases the material must also be fully dense. To achieve ceramics with a fine enough grain size requires the use of nanopowders. There are numbers of different wet chemical methods to synthesis nanocrystalline YAG powders with high purity, but only a few yield highly enough quality powders. Routes such as sol-gel $[6,7]$, co-precipitation $[6,8]$, and combustion synthesis $[9,10]$, often produce single phase YAG powders, but with a high degree of agglomeration. The latter occurs during the drying and calcination stages, which are usually 
essential to convert the precursors into crystalline powders. Therefore, a route is required in which crystallisation occurs whilst the nanoparticles are suspended in a liquid medium. Supercritical fluids offer such opportunities, for example, they have been used to crystallise metal-organic or inorganic precursors into metal oxides. This route also avoids the high temperature heat treatments usually required [11].

Cabanas and co-workers [12] produced single phase YAG in supercritical ethanol and water mixtures using continuous synthesis. The solvent mixtures facilitated the crystallization of the precipitates obtained using ammonia solution at $385^{\circ} \mathrm{C}$ and 24 $\mathrm{MPa}$. Zhang et al. [13] reduced the processing conditions to $290^{\circ} \mathrm{C}$ and $10 \mathrm{MPa}$ in a batch type reactor by using a 2:1 ethanol-water mixture, nitrate precursors and ammonium hydrogen carbonate as the precipitant. They obtained monodispersed, spherical particles with a mean size of $\sim 60 \mathrm{~nm}$.

Inoue et al. [14] reported the solvothermal synthesis of YAG using organic precursors such as yttrium acetate and aluminium isopropoxide. They investigated ethylene glycol and 1, 4 butanediol as the solvent medium; the former resulted in amorphous YAG whilst the latter yielded phase-pure crystalline YAG consisting of 30 $\mathrm{nm}$ agglomerates of $10 \mathrm{~nm}$ primary particles after achieving crystallisation by holding at $300^{\circ} \mathrm{C}$ and $5 \mathrm{MPa}$ for two hours. The method was also successfully used by Kasuya et al. [15] and Nyman et al. [16] to produce well-crystallized, but agglomerated, YAG:Ce ${ }^{3+}$ nanophosphor powder by adding cerium (III) acetate to the reaction mixture. Kasuya et al. [17] further investigated the reaction rate and found that a holding time of less than one hour was insufficient to achieve crystallisation.

Thus the major problem with the nano YAG powders produced to date has been the level of agglomeration present and the lack of control over it. The present work was 
therefore undertaken with a view to avoiding or minimising this problem and different analytical methods were used to gain an insight into the formation mechanism and growth kinetics involved when 1, 4 butanediol was used as the solvent.

\section{Experimental}

The organic precursors used were $99.9 \%$ pure $0.6 \mathrm{M}$ yttrium acetate hydrate $\left(\mathrm{Y}\left(\mathrm{CH}_{3} \mathrm{CO}_{2}\right)_{3} \cdot \mathrm{xH}_{2} \mathrm{O}\right)$ and $\geq 98 \%$ pure $1 \mathrm{M}$ aluminium isopropoxide $\left(\mathrm{Al}\left(\mathrm{OCH}\left(\mathrm{CH}_{3}\right)_{2}\right)_{3}\right)$ both from Sigma Aldrich, Dorset, UK. These provided the source of $\mathrm{Y}^{3+}$ and $\mathrm{Al}^{3+}$ ions respectively. 99.9\% pure 1 M 1, 4 butanediol, also from Sigma Aldrich, was used as the solvent medium. A conventional autoclave reactor from Prosense, Munich, Germany, rated to $400^{\circ} \mathrm{C}$ and $10 \mathrm{MPa}$ (for safety reasons, $8.5 \mathrm{MPa}$ was set as the maximum working limit) was used for the synthesis.

Table 1 Experimental conditions investigated for synthesis of nano YAG

\begin{tabular}{|c|c|c|c|}
\hline \multirow{2}{*}{ Autogenous Conditions } & \multicolumn{3}{|c|}{ Volume of the precursor used in a $400 \mathrm{ml}$} \\
\cline { 2 - 4 } & 1,4 butanediol & Yttrium & Aluminium \\
& & acetate & isopropoxide \\
\hline $270^{\circ} \mathrm{C}$ for $2 \mathrm{~h}$ and $2.0 \mathrm{MPa}$ & $176 \mathrm{ml}$ & $8.48 \mathrm{~g}$ & $8.52 \mathrm{~g}$ \\
\hline $280^{\circ} \mathrm{C}$ for $2 \mathrm{~h}$ and $6.0 \mathrm{MPa}$ & $176 \mathrm{ml}$ & $8.48 \mathrm{~g}$ & $8.52 \mathrm{~g}$ \\
\hline $300^{\circ} \mathrm{C}$ for $2 \mathrm{~h}$ and $8.5 \mathrm{MPa}$ & $176 \mathrm{ml}$ & $8.48 \mathrm{~g}$ & $8.52 \mathrm{~g}$ \\
\hline $300^{\circ} \mathrm{C}$ for $2 \mathrm{~h}$ and $7.0 \mathrm{MPa}$ & $213 \mathrm{ml}$ & $10.29 \mathrm{~g}$ & $10.34 \mathrm{~g}$ \\
\hline $300^{\circ} \mathrm{C}$ for $2 \mathrm{~h}$ and $6.0 \mathrm{MPa}$ & $249 \mathrm{ml}$ & $12.00 \mathrm{~g}$ & $12.06 \mathrm{~g}$ \\
\hline
\end{tabular}


A stoichiometric ratio of $3 \mathrm{Y}$ to $5 \mathrm{Al}$ was prepared by mixing appropriate amounts of the two precursors, weighed to two decimal places, in the butanediol solvent, Table 1. The mixture was placed in a $400 \mathrm{ml}$ autoclave vessel and processed at different temperatures and autogenous pressures, see Table 1, the volume of the precursor adjusted to achieve the desired pressure. The resultant white suspension was centrifuged at $3500 \mathrm{rpm}$, washed three times using ethanol to remove the precursor residues, and then dried at $60^{\circ} \mathrm{C}$ for 24 hours before being ground using an agate pestle and mortar for at least $1 \mathrm{~min}$.

Fourier transform-infrared spectroscopy (FT-IR 8400S, Shimadzu, Maryland, USA) was performed to investigate the functional groups present in the suspensions obtained from the synthesis route before drying. A drop of the suspension was placed between two $\mathrm{NaCl}$ discs, forming a thin film, and the transmittance of the suspensions studied over the range $4000-400 \mathrm{~cm}^{-1}$. The degree of crystallinity and phase(s) present were determined using an X-ray diffractometer (Bruker D8, Bruker AXS GmbH, Karlsruhe, Germany) at a step size of $0.02^{\circ}$ from $20^{\circ}$ to $90^{\circ} 2 \theta$. The average crystallite size was calculated from the line broadening of (321), (400), (420), (422), (431) and (521) reflections using the Scherrer formula reported elsewhere $[8,10]$.

The morphology and the particle size of the powders were also determined using transmission electron microscopy, TEM (JEOL JEM 2000FX, JEOL Ltd. Tokyo, Japan). For this a 5 wt.\% suspension of the powder dispersed in methanol using 3 minutes of ultrasound (Soniprep 150-MSE ultrasonicator, MSE Scientific Instruments, Manchester, UK) was deposited on a carbon coated copper grid (Agar Scientific, Stansted, UK) and dried in an oven at $60^{\circ} \mathrm{C}$ for 15 minutes before loading 
into a single tilt holder. The TEM was operated at $200 \mathrm{kV}$. A different TEM, operated in STEM mode (TechnaiF20, FEI, Hillsboro, OR, USA), was used to obtain the lattice fringes of the particles. Sample preparation was identical to that just described, but the sample was dried in UV light for 1 hour rather than in oven to avoid breaking the carbon film. This TEM was also operated at $200 \mathrm{kV}$ and images were obtained using a CCD camera.

The BET surface area, S, of the YAG nanoparticles was measured using an automated gas adsorption analyser (Tristar ${ }^{\mathrm{TM}} 3000$ Micrometrics Instrument Corporation, Norcross, GA, USA). Equation 1 [18] was used to obtain the mean particle size from the surface area data. Comparison of the data from the TEM and BET provided an insight into the extent of agglomeration of the particles.

$$
\text { Particle size }\left(d_{B E T}\right)=\frac{6000}{\rho \times S} \quad \text { Eqn } 1
$$

where, $\mathrm{S}$ is the specific surface area $\left(\mathrm{m}^{2} \mathrm{~g}^{-1}\right)$ and $\rho$ is the theoretical density $(4.55 \mathrm{~g}$ $\mathrm{Cc}^{-1}$ ) of the material.

After centrifuging the suspension at 5000 rpm for 10 mins (Heraeus Centrifuge, Osterode, Germany), the supernatant was analysed using gas chromatography mass spectroscopy, GC-MS (model HP-6890 with HP5973, mass selective detector, Hewlett Packard, Anaheim, USA). The GC-MS was also equipped with a flame ionisation detector, which was connected with a $30 \mathrm{~m}$ capillary column (DB-WAX J\&W Scientific, Santa Clara, USA). Each sample was diluted with chloroform in a 1:10 ratio and $\sim 4 \mu \mathrm{l}$ injected for each analysis. The temperature, for both the injector and the detector, was set at $300^{\circ} \mathrm{C}$ and held for 10 mins to desorb all the organics. Helium was used as a carrier gas at a continuous flow rate of $1.0 \mathrm{ml} \mathrm{min}^{-1}$. Data on 
the chemical compounds present was identified based on the $\mathrm{GC}$ retention time on the column, the total time taken for each sample was 46.5 mins, and the matching of mass spectra with NIST standard reference databases such as Mainlib and Replib [19-21].

\section{Results and Discussion}

\subsection{Powder characteristics}

The XRD patterns, Figure 1, revealed that single phase YAG, exactly matching the 33-0040 JCPDS YAG reference, was achieved using all the temperatures investigated, $270^{\circ} \mathrm{C}-300^{\circ} \mathrm{C}$, and their respective autogenous pressures. The primary differences between the different samples were the degree of crystallinity (see the peak intensities) and the crystallite sizes (21.7 nm to $22.4 \mathrm{~nm}$, see table 2) calculated using the Scherer's line broadening method, which increased with increasing temperature and pressure as expected. No intermediate phases, such as YAH, YAM or YAP, were observed in any of the samples prepared. These results suggest that the use of highly reactive 1,4 butanediol caused crystallisation into the YAG structure under conditions well below those required for supercriticality of the solvent $\left(-454^{\circ} \mathrm{C}\right.$ and $\left.6.2 \mathrm{MPa}\right)$ [22]. This suggests that the organic precursors underwent complete dissolution and crystallised into single phase YAG, within the detection limit of XRD. 


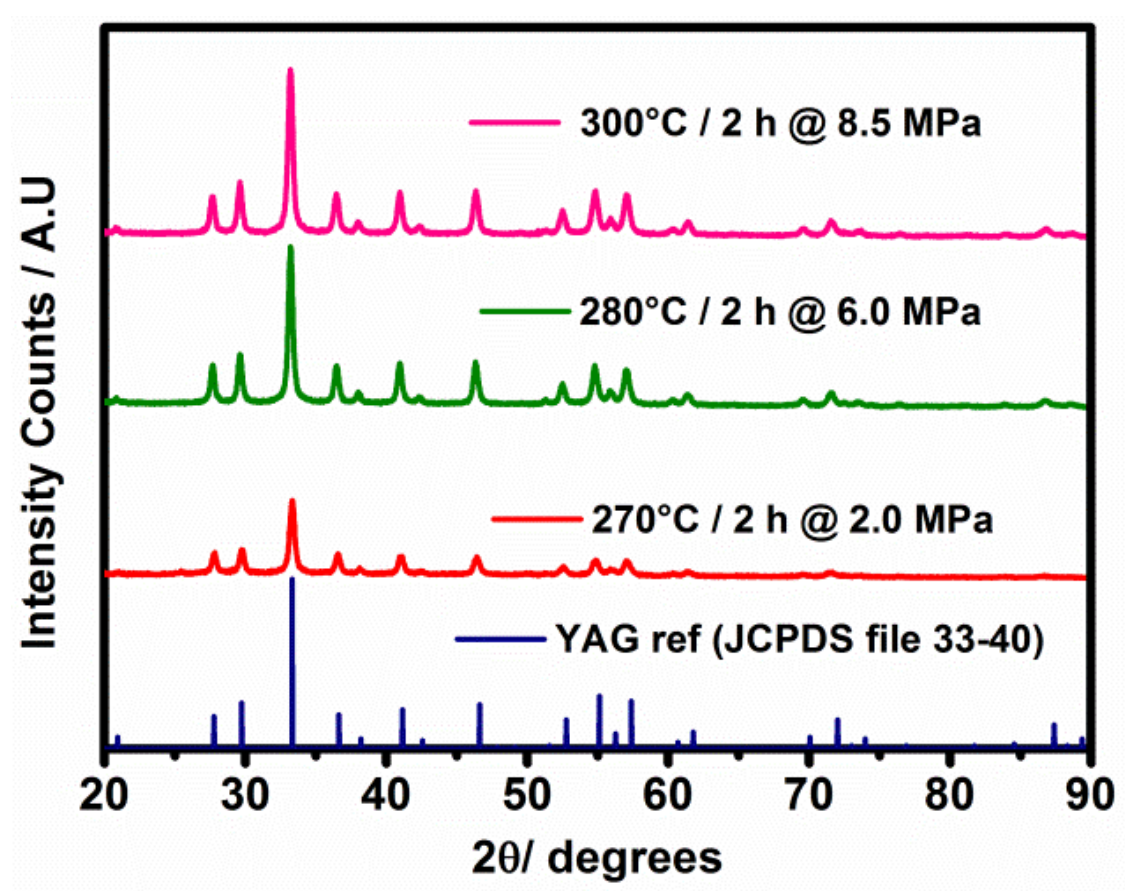

Figure 1 XRD patterns of solvothermal nano YAG powders obtained using 1, 4 butanediol under the conditions indicated.

\subsection{Formation mechanism}

The key to the formation of the YAG particles and their growth kinetics can be attributed to the transformation of 1,4 butanediol during the reaction. It has been reported that it transforms into tetrahydrofuron when the temperature and pressure exceed $250^{\circ} \mathrm{C}$ and $18 \mathrm{MPa}$ [24]. In the present case, where the temperature exceeded the critical value but the pressure did not, the functional groups from the precursors were suspected to have reacted with the butanediol derivatives, possibly leading to a different reaction pathway (see GC-MS data) compared with the normal tetrahydrofuron transformation. 




\begin{tabular}{|c|c|c|}
\hline Peak no & $\begin{array}{c}\text { Peak } \\
\text { position }\end{array}$ & Description \\
\hline 1 & $\sim 3296$ & $\mathrm{v}(\mathrm{OH})$ \\
\hline 2 & $\sim 2936$ & $\mathrm{v}_{\mathrm{s}}\left(\mathrm{CH}_{2}\right)$ \\
\hline 3 & $\sim 2868$ & $\mathrm{v}_{\mathrm{as}}\left(\mathrm{CH}_{2}\right)$ \\
\hline 4 & $\sim 1725$ & $\mathrm{v}_{\mathrm{s}}(\mathrm{C}=\mathrm{O})$ \\
\hline 5 & $\sim 1650$ & $\mathrm{v}_{\mathrm{as}}(\mathrm{C}=\mathrm{O})$ \\
\hline 6 & $\sim 1580$ & $\mathrm{v}(\mathrm{C}=\mathrm{O})$ \\
\hline 7 & $\sim 1460$ & $\delta_{\mathrm{s}}\left(\mathrm{CH}_{2}\right)$ \\
\hline 8 & $\sim 1430$ & $\delta_{\mathrm{as}}\left(\mathrm{CH}_{2}\right)$ \\
\hline
\end{tabular}

Figure 2 : FT-IR spectroscopy of the precursor sol compared with the suspension processed under conditions indicated and the assignment of the peaks, after $[16,25]$.

The FT-IR spectra and with the corresponding peak assignments for the precursor sol, a mixture of YAG precursors and 1, 4 butanediol solvent, along with the assynthesised YAG suspensions processed at $270^{\circ} \mathrm{C} \& 2 \mathrm{MPa}$ for $2 \mathrm{~h}$ and $300^{\circ} \mathrm{C} \& 8.5$ $\mathrm{MPa}$ for $2 \mathrm{~h}$, are shown in Figure 3. It is clear from the spectra that the peaks corresponding to the hydroxyl vibration, $\mathrm{v}(\mathrm{OH})$, from the 1, 4 butanediol and aluminium isopropoxides overlapped at around $\sim 3296 \mathrm{~cm}^{-1}$. This peak, labelled 1, became broader after processing, which could be attributed to the dehydration of $\mathrm{OH}^{-}$ions from the 1, 4 butanediol [25]. The doublet peaks obtained near $\sim 2936 \mathrm{~cm}^{-1}$ ( $2 \& 3$ ) and $\sim 1450 \mathrm{~cm}^{-1}(7 \& 8)$, the characteristic peaks of 1,4 butanediol, are the symmetric and asymmetric modes of stretching and bending vibrations of $\left(\mathrm{CH}_{2}\right)$, respectively [16]. These peaks were obtained in all the spectra suggesting that the 
carbon chain was not broken, whilst the $\mathrm{OH}^{-}$ions on either side of the butanediol $\left(\mathrm{OH}-\mathrm{CH}_{2}-\mathrm{CH}_{2}-\mathrm{CH}_{2}-\mathrm{OH}\right)$ were dehydrated.

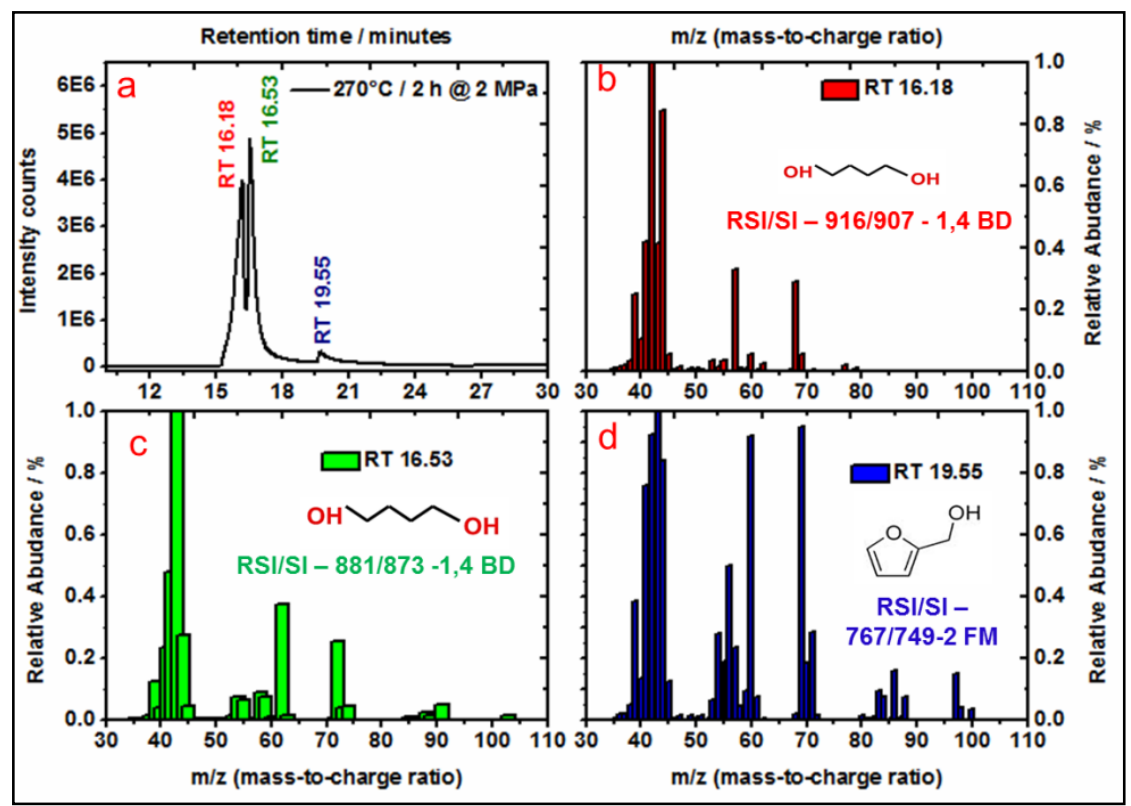

Figure 3 : GC-MS results of the as-synthesised suspension obtained after processing the precursor at $270^{\circ} \mathrm{C}$ and $2 \mathrm{MPa}$ for $2 \mathrm{~h}$; (a) the GC spectra; (b), (c) and (d) are the respective MS spectra of the peaks obtained at retention times of $16.18,16.53$ and 19.55 mins, respectively.

The peak in the precursor sol at $\sim 1580 \mathrm{~cm}^{-1}$ (6) is the stretching vibration of the $v(C=0)$ molecule from the yttrium acetate [16]; it has been shifted into a doublet (4 \& 5) as symmetric and asymmetric vibrations from $\sim 1725-1650 \mathrm{~cm}^{-1}$ in the assynthesised YAG suspensions. This suggests that the acetate group has been cleaved from the yttrium acetate and formed a new chemical compound. Since identification of the compound could provide valuable information about the chemical reaction leading to the formation of YAG nanoparticles, GC-MS was undertaken. 


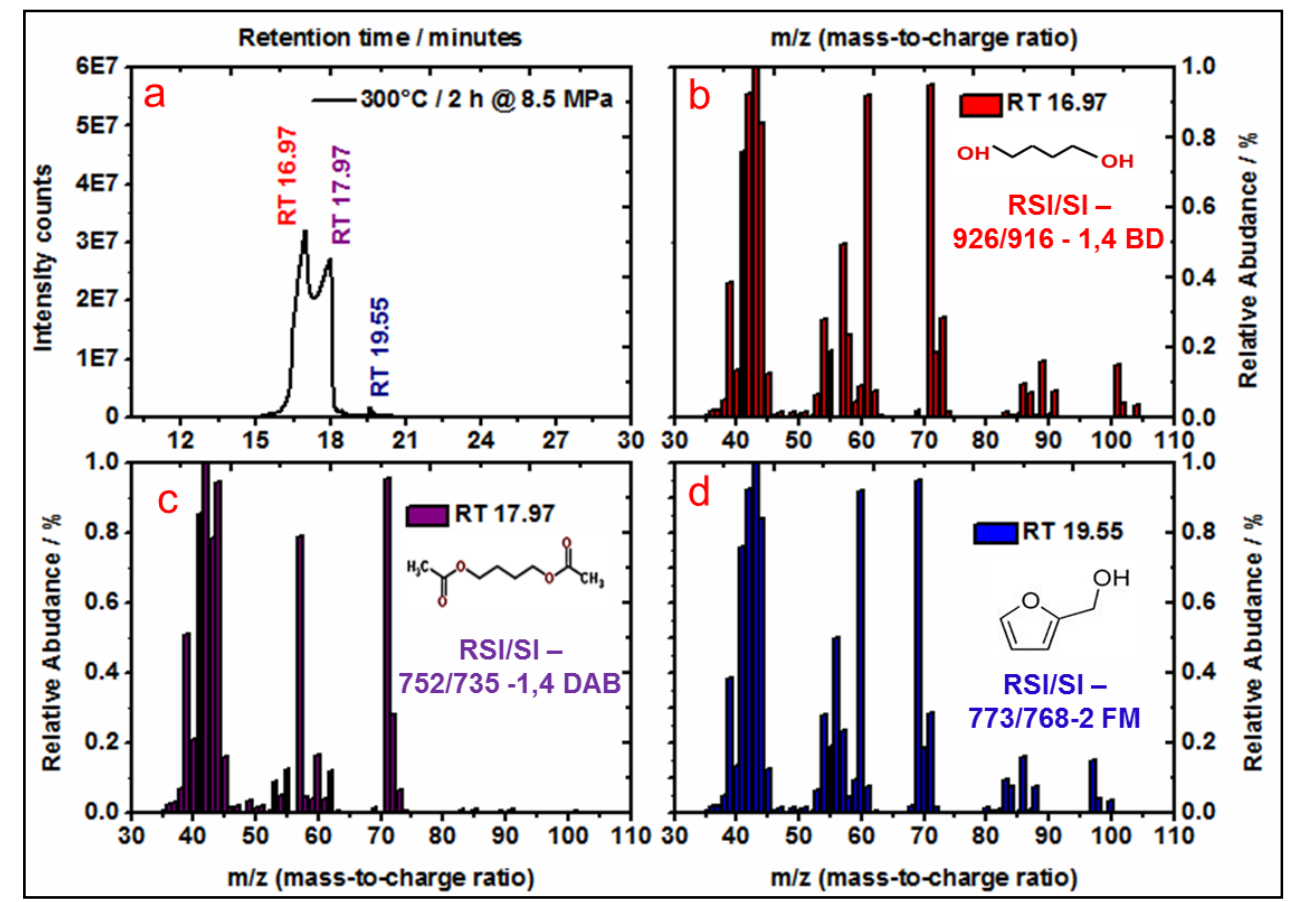

Figure 4 : GC-MS results of (a) the as-synthesised suspension obtained after processing the precursor at $300^{\circ} \mathrm{C}$ and $8.5 \mathrm{MPa}$ for $2 \mathrm{~h}$. The GC spectra (b), (c) and (d) are the respective MS spectra of the peaks obtained at retention times of $16.97,17.97$, and 19.55 mins, respectively.

The spectra obtained for the suspension processed at $270^{\circ} \mathrm{C}$ and $2 \mathrm{MPa}$ for $2 \mathrm{~h}$ showed a small amount of 2-furan methanol (2-FM) at $19.55 \mathrm{~min}$ and the major peaks corresponding to 1,4 butanediol at 16.18 and $16.53 \mathrm{~min}$, Figure 4 . The compounds were identified based on the GC retention time on the column and the computer matching of mass spectra results of the respective peaks with standards [19]. This suggested that the dehydration had been initiated but was not significant. The dehydrated 1, 4 butanediol then reacted with the terminal alcohol groups from the aluminium isopropoxide to form the 2-FM, Figure $4 \mathrm{~d}$.

When the butanediol and the precursor mixture was subjected to $300^{\circ} \mathrm{C}$ and $8.5 \mathrm{MPa}$ for $2 \mathrm{~h}$, the result was $2 \mathrm{FM}, 1,4$ diacetoxybutane (1, 4 DAB) and 1, 4 butanediol 
with these occurring at 19.55, 17.97 and 16.97 min, respectively, Figure 5. The partially dehydrated 1, 4 butanediol will have reacted with the terminal acetate groups from the yttrium acetate and undergone esterification to form the 1, 4 DAB [26], a reaction that will have influenced the crystallisation and growth kinetics of the nanoparticles. Figure 6 shows the proposed reaction mechanism. It is believed that a parallel, catalytic chemical reaction took place between the precursors and the solvent. The XRD results showed that the organic precursors reacted to form YAG nanoparticles, whilst the GC-MS revealed that the 1, 4 butanediol reacted with the chemical species of acetate and alcohol to form 1, 4 DAB and 2-FM. It has been reported that metal oxides act as catalysts to dehydrate alcohols and diols into olefins and unsaturated alcohols, respectively, and that the rate of the reaction depends on the size of the oxide particles being used [27]. These catalytic reactions are often promoted by both structure-sensitive and structure-insensitive reactions and most of the metal oxides underwent the structure-sensitive reaction [28]. Therefore, chemical reaction and particle growth are interdependent in these processes; one influences the other significantly. For instance, Wang et al. [29] witnessed particle growth under hydrothermal conditions along a preferential orientation for ceria nanoparticles. They concluded that the coordinated uncapped particles matched with their lattice planes with similar Miller indices and grew into large particles by minimizing their interfacial energies. Meanwhile, Igarashi et al. [30] investigated the dehydration of 1,4 butanediol into unsaturated alcohols using $\mathrm{CeO}_{2}$ catalysts with different particle sizes. They observed that the catalytic performance in the cyclisation of butanediol into tetrahydrofuran and $\mathrm{y}$-butyrolactone was greatly influenced by the size of the ceria particles. These studies suggest that particle 
growth occurs at the preferential lattice planes and particle size is very sensitive to changes in chemical kinetics during solvothermal reactions.

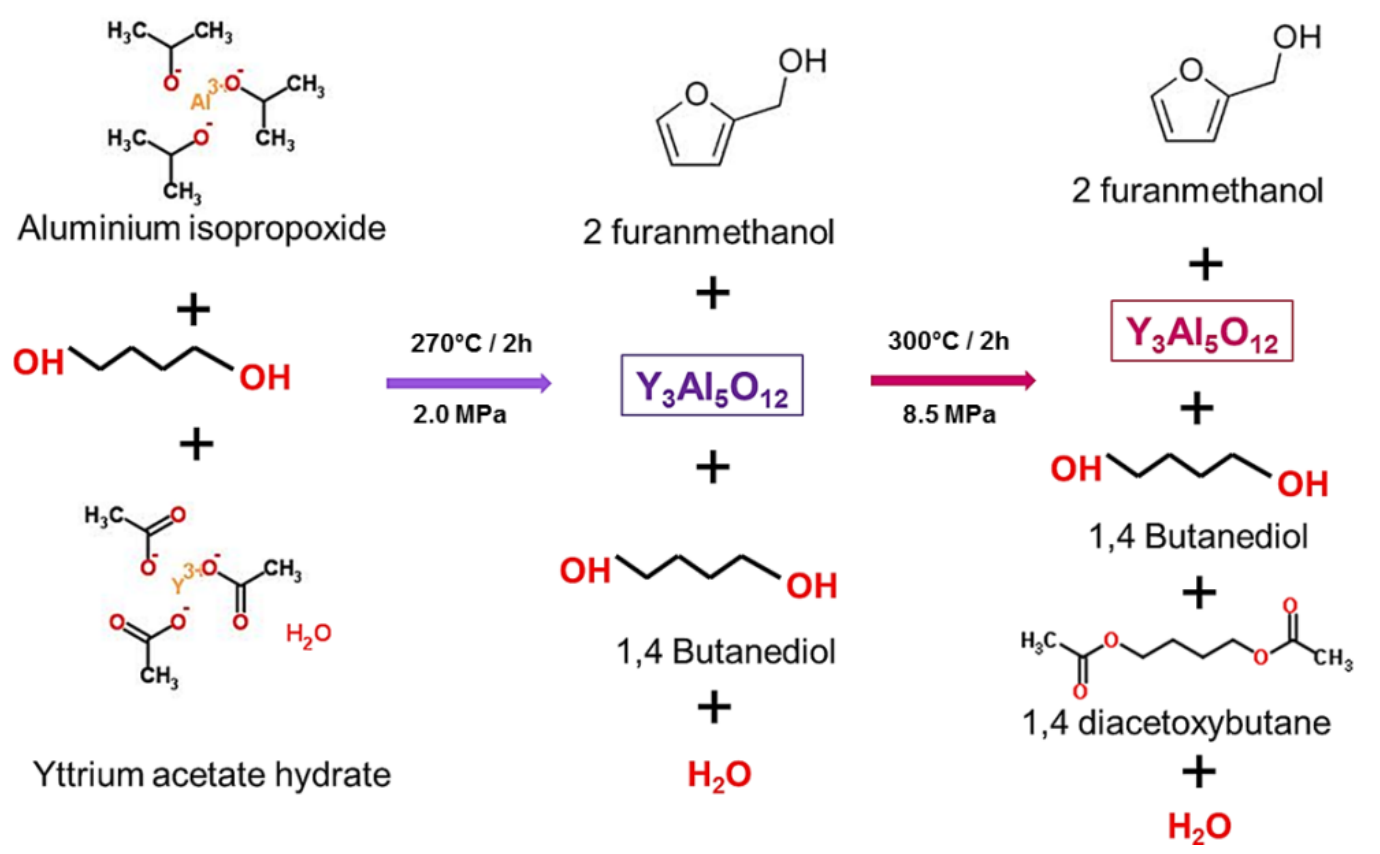

Figure 5 Proposed reaction mechanism underpinning solvothermal synthesis of YAG using 1,4 butanediol

\subsubsection{Particle growth kinetics}

To gain an insight into the particle growth kinetics and the extent of agglomeration under different synthesis conditions, the numerical values of the particle size calculated from the XRD spectra and the specific surface area from BET have been compared with the particle sizes estimated using TEM, see table 2. It is clear that the crystallite sizes $\left(D_{X R D}\right)$ of the nano YAG powders from all the conditions used were relatively lower than the values estimated from the BET and TEM data. This is due to the fact that the Scherer equation doesn't take account of instrument broadening errors and the XRD only measures average volume of the coherent/uniform regions where the particles are highly crystalline [23], whereas the BET and TEM techniques 
use surface area and 2 dimensional information for the size and shape of the particles, respectively.

Table 2 Comparison of the YAG particle sizes estimated using different techniques

\begin{tabular}{|c|c|c|c|c|}
\hline Conditions & $\mathrm{D}_{\mathrm{XRD}} / \mathrm{nm}$ & $\mathrm{D}_{\mathrm{BET}} / \mathrm{nm}$ & $\mathrm{D}_{\mathrm{TEM}} / \mathrm{nm}$ & $\begin{array}{c}\text { BET surface } \\
\text { area } / \mathrm{m}^{2} \mathrm{~g}^{-1}\end{array}$ \\
\hline $270^{\circ} \mathrm{C} / 2 \mathrm{~h} @ 2.0 \mathrm{MPa}$ & $21.7 \pm 2.2$ & $27.8 \pm 0.4$ & $7 \pm 1(27 \pm 3)^{\star}$ & $46.8 \pm 1$ \\
\hline $280^{\circ} \mathrm{C} / 2 \mathrm{~h} @ 6.0 \mathrm{MPa}$ & $24.7 \pm 0.7$ & $28.0 \pm 0.3$ & $28 \pm 3$ & $44.1 \pm 1$ \\
\hline $300^{\circ} \mathrm{C} / 2 \mathrm{~h} @ 8.5 \mathrm{MPa}$ & $22.4 \pm 0.7$ & $27.9 \pm 0.4$ & $27 \pm 3$ & $46.7 \pm 1$ \\
\hline
\end{tabular}

*agglomerate size measured from TEM

However under the synthesis conditions of $270^{\circ} \mathrm{C}$ and $2 \mathrm{MPa}$, Figure $2 \mathrm{a}$, the primary particle size determined from TEM was about $7 \pm 1 \mathrm{~nm}$, but the morphology clearly reveals that the primary particles were agglomerated into $\sim 27 \mathrm{~nm}$ clusters.. On the other hand, the particle sizes calculated from XRD and BET analyses suggest that both the techniques considering the clusters as individual particles; this is because the former cannot distinguish the boundaries in the clusters [31], whilst in the BET technique the nitrogen cannot permeate further into the clusters to classify the primary particles seen through TEM [32]. At $280^{\circ} \mathrm{C}$ the autogenous pressure increased to $6 \mathrm{MPa}$ and the resultant primary particles were coarser, $\sim 28 \mathrm{~nm}$, with an irregular structure and, in some instances, clusters were noticed from TEM, Figure $2 \mathrm{~b}$. This effect has also been supplemented by the XRD and BET results. On further increase in temperature to $300^{\circ} \mathrm{C}$, the autogenous pressure increased to 8.5 $\mathrm{MPa}$ and homogenous spherical nanoparticles were formed also measuring $\sim 27 \mathrm{~nm}$ from TEM, Figure 2c. The latter particles had a surface area of about $46.7 \pm 1 \mathrm{~m}^{2} \mathrm{~g}^{-1}$, yielding a calculated BET particle size from equation 1 of $\sim 27 \mathrm{~nm}$ and the calculated 
$D_{X R D}$ was about 22.4. Further, the $D_{B E T}$ matches the $\sim 27 \mathrm{~nm}$ diameter obtained from the TEM study surprisingly well and supports the $D_{\text {TEM }}$ data that the particles obtained using solvothermal process exhibited relatively little agglomeration.

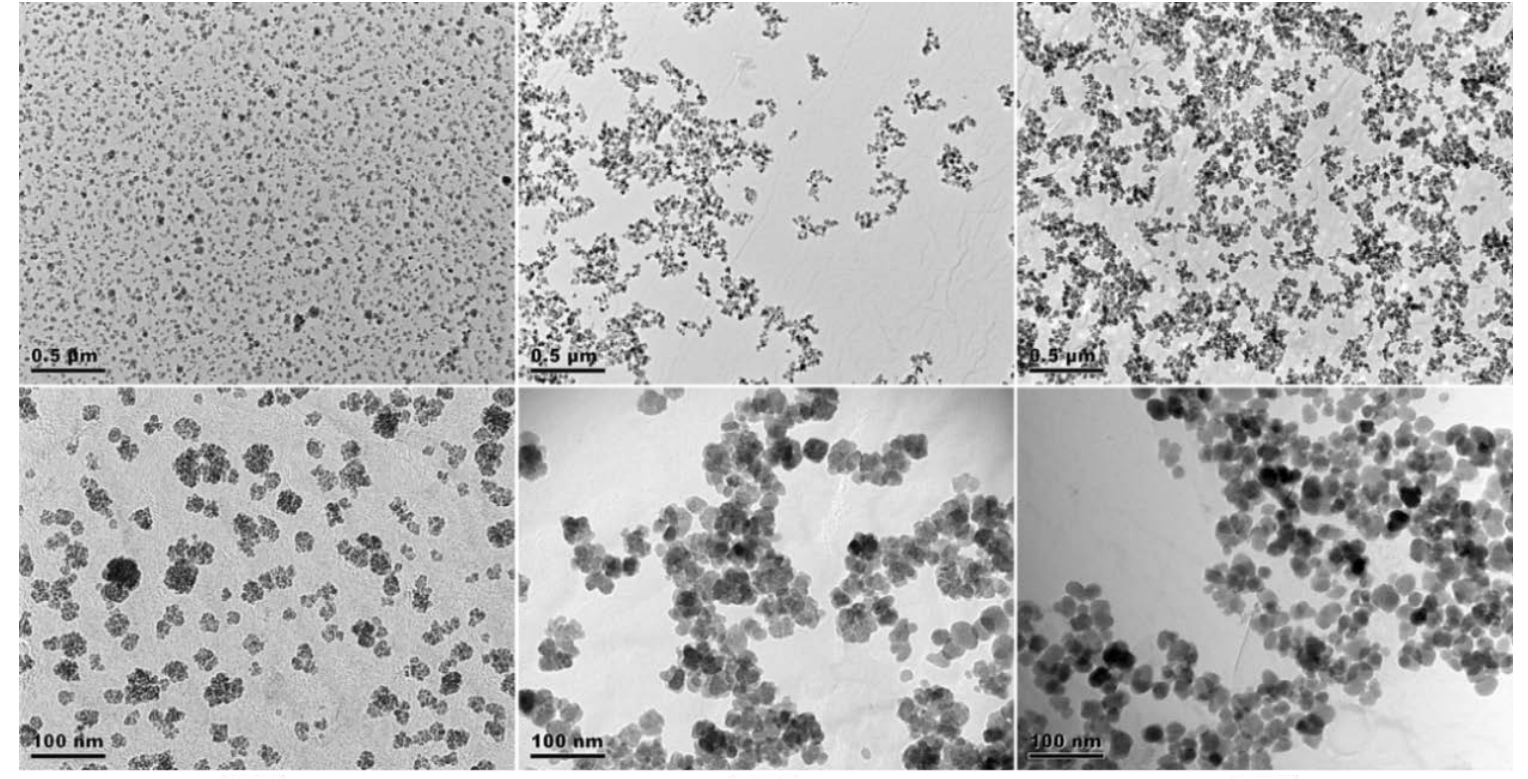

(a) (b) (c)

Figure 6 : TEM images of solvothermal nanoYAG powders obtained using 1, 4 butanediol under the conditions (a) $270^{\circ} \mathrm{C} / 2 \mathrm{~h} @ 2 \mathrm{MPa}$ (b) $280^{\circ} \mathrm{C} / 2 \mathrm{~h} @ 6 \mathrm{MPa}$ and (c) $300^{\circ} \mathrm{C} / 2 \mathrm{~h} @ 8.5 \mathrm{MPa}$

To understand the growth kinetics further, different volumes of precursors, see Table 1, with same stoichiometry were processed in the autoclave to achieve different pressures at $300^{\circ} \mathrm{C}$; all the particles obtained showed similar morphology, Figure 7 . Although most of the particles were similar in size after synthesising at $300^{\circ} \mathrm{C}$ and 6 $\mathrm{MPa}$, some clusters of finer $7 \pm 1 \mathrm{~nm}$ particles were still present; arrows indicate these in Figure 7a. Less evidence of these particles was observed when the pressure was $7 \mathrm{MPa}$ or higher and the particles formed were very homogenous in both size and morphology. This is in accordance with the particle morphologies of Cerium doped YAG synthesised by Nyman [16] and Kasuya [15] using the same 
precursors as in the present work, except dopant. Kasuya used $220^{\circ} \mathrm{C}$ for $4-14$ days (no mention of the autogenous pressure) whilst Nyman used at $300^{\circ} \mathrm{C}$ for $4 \mathrm{~h}$ and an autogenous pressure of 5.5 MPa. Regardless of the temperature and time, both their results showed $\sim 27 \mathrm{~nm}$ clusters of $7 \pm 1 \mathrm{~nm}$ primary particles and clearly revealed that the particle growth kinetics were enhanced by pressure. The current STEM analysis supports this result.

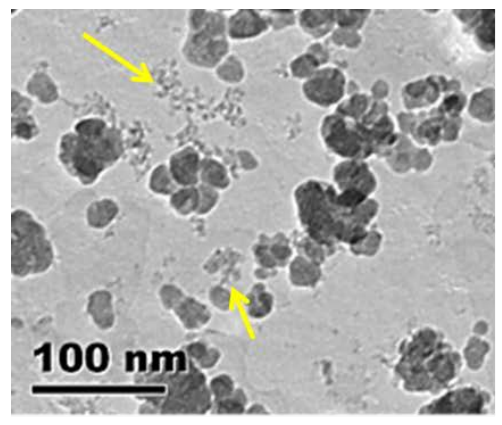

(a)

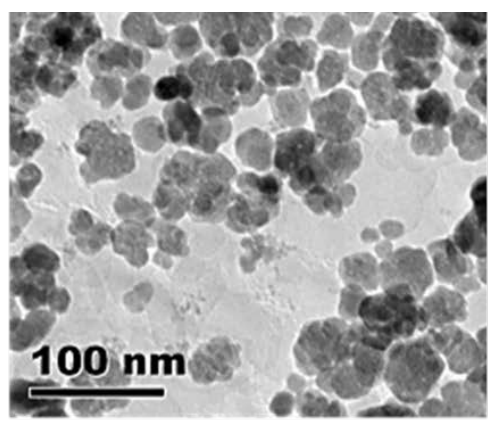

(b)

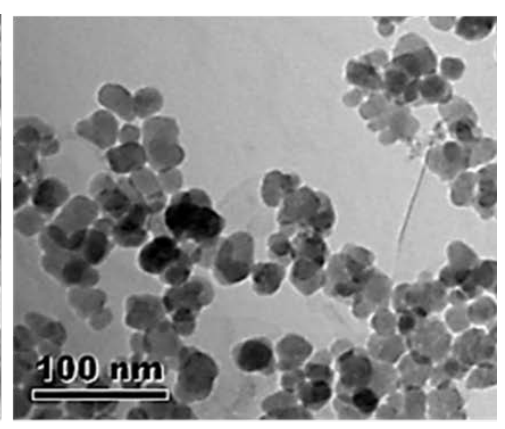

(c)

Figure 7 Effect of pressure on ST-nYAG particles at $300^{\circ} \mathrm{C}$ for $2 \mathrm{~h}$ (a) $6 \mathrm{MPa}$, (b) 7 $\mathrm{MPa}$ and (c) $8.5 \mathrm{MPa}$. Arrows in (a) show the presence of small particles mentioned in the text.

A proposed particle growth mechanism is illustrated in Figure $8 \& 9$ based on the STEM results. The nanoparticles synthesised at $270^{\circ} \mathrm{C}$ and $2 \mathrm{MPa}$ for $2 \mathrm{~h}$ were agglomerated by oriented attachment (OA), a process that causes neighbouring particles to assemble in order to share a common crystallographic orientation. This yields facile coalescence [33-35], Figure 8a. The parallel lattice fringe spaces were about $0.49 \mathrm{~nm}$ apart, Figure 9, a value that is closely associated with $\{112\}$ facets of the (211) atomic planes of YAG [36,37]. These planes exhibit high surface energy compared to the other low-index planes of YAG [37,38]. Qin et al. [37] suggested a growth mechanism based on the crystallographic orientation of YAG particles, they thought that the particle growth kinetics occurred via a self-assembled, Ostwald 
ripening process at different calcination temperatures. They found that the fine YAG particles coalesced into coarser particles by reducing their total interfacial energy along the $<112>$ crystallographic direction of the $\{112\}$ planes. Very similar behaviour was observed here, suggesting that the $\{112\}$ facets are the active site favouring particle growth for YAG. Having said this, the low diffraction intensity in the XRD data implies that the degree of crystallinity is relatively weak.

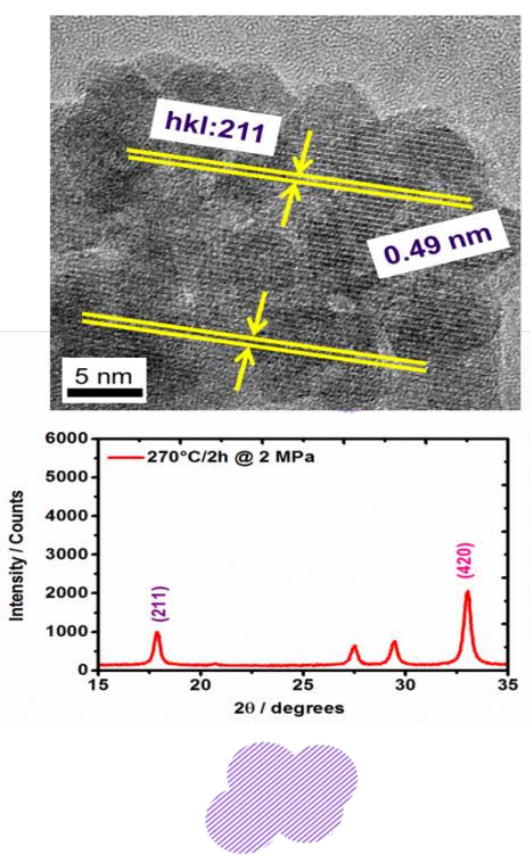

hkl:211

(a)
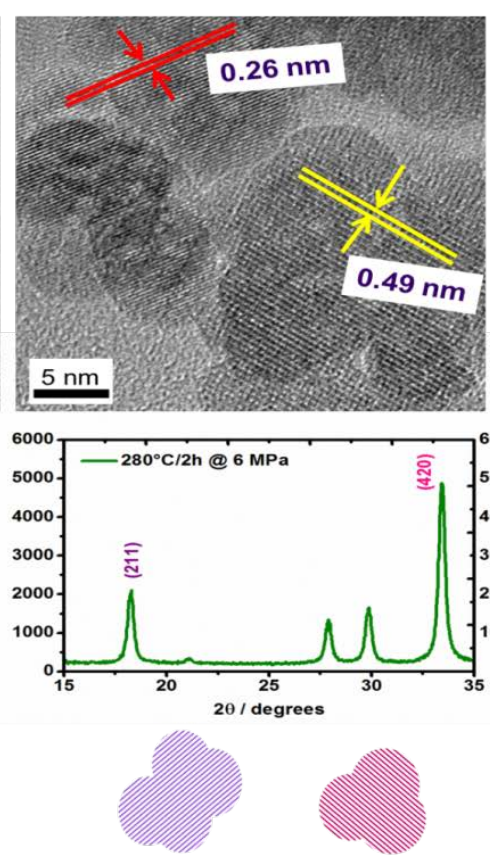

hkl:420
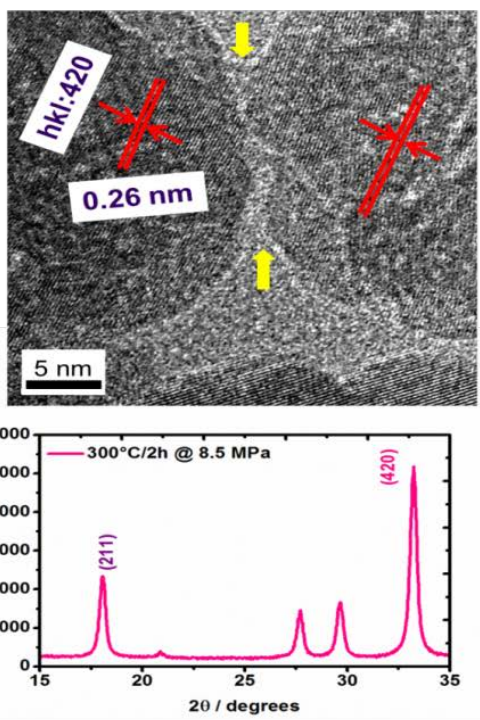

(b)

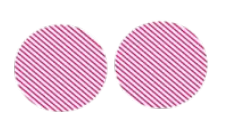

hkl:420

(c)

Figure 8 YAG growth mechanism: STEM images (top) and XRD (middle) with a schematic of particles (bottom) obtained under three different conditions (a) $270^{\circ} \mathrm{C}$ and $2 \mathrm{MPa}$ for $2 \mathrm{~h}$, (b) $280^{\circ} \mathrm{C}$ and $6 \mathrm{MPa}$ for $2 \mathrm{~h}$ and (b) $300^{\circ} \mathrm{C}$ and 8.5 MPa for $2 \mathrm{~h}$

When the synthesis conditions were increased to $280^{\circ} \mathrm{C}$ and $6 \mathrm{MPa}$ for $2 \mathrm{~h}$, the XRD result showed a substantial increase in the intensity of the data, Figure 8b, which implies that the degree of crystallinity was improved significantly. The STEM image displayed two different (hkl) planes, (211) and (420), which suggests that the 
agglomerated particles with (211) planes underwent internal structural rearrangement of yttrium and aluminium atoms $(0.23 \mathrm{~nm}$ and $0.18 \mathrm{~nm}$ radii, respectively [39]) due to atomic diffusion and therefore forming particles with a (420) plane arrangement.
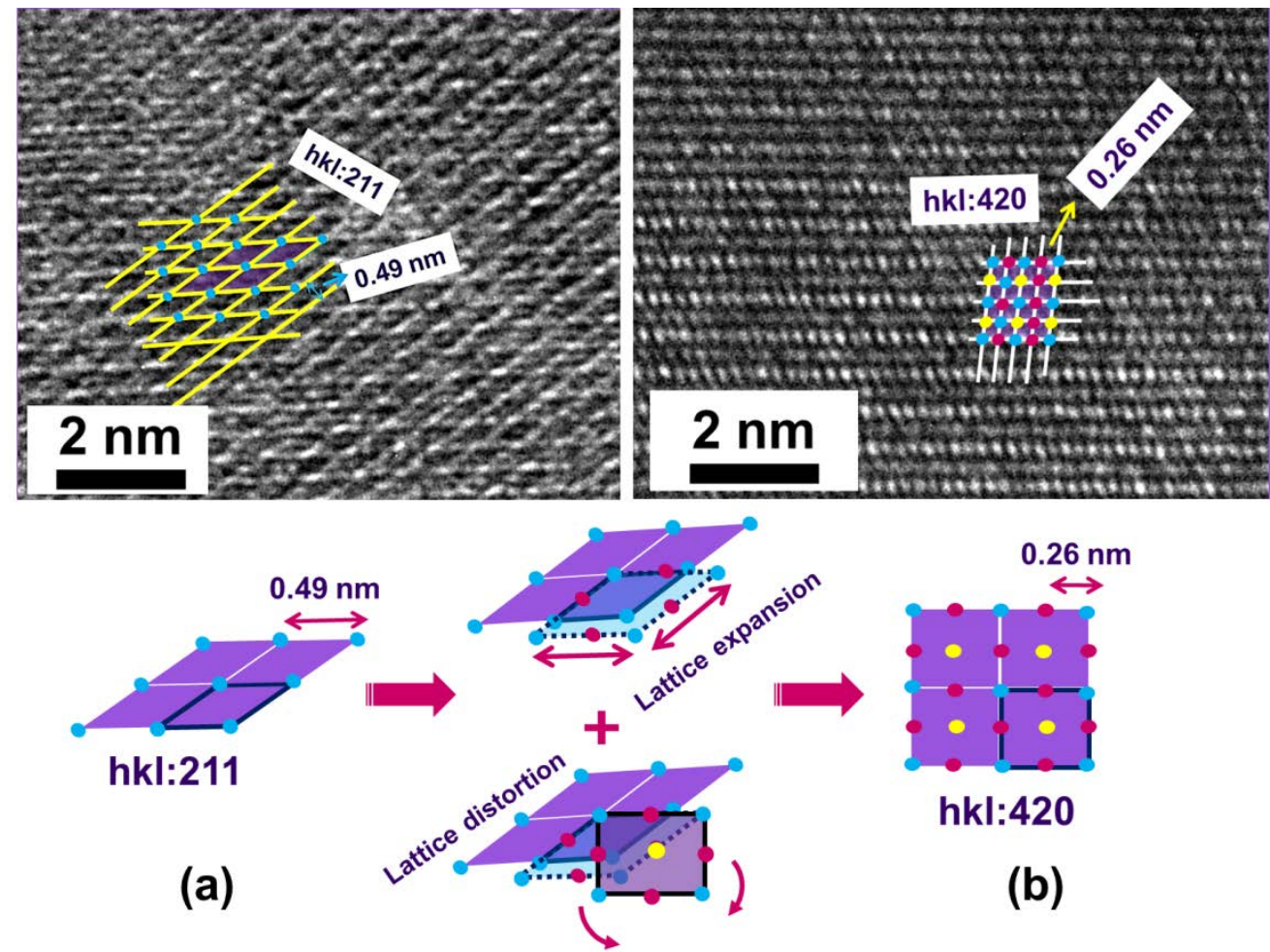

(b)

Figure 9 Top, Lattice fringes of (211) and (420) planes obtained at high magnification using STEM and below, schematic illustration of atomic rearrangement. Particles obtained at (a) $270^{\circ} \mathrm{C}$ and $2 \mathrm{MPa}$ for $2 \mathrm{~h}$ and (b) $300^{\circ} \mathrm{C}$ and $8.5 \mathrm{MPa}$ for $2 \mathrm{~h}$

The schematic shown in Figure 9 illustrates the atomic rearrangement by lattice distortion and expansion. The distortion is usually due to invariant lattice shear caused by bilateral tilting and rotation of atoms [40], which could have happened due to the use of higher pressure. This result in cationic displacements [41] within the planes caused by interstitial and substitution mechanisms, which will have influenced lattice expansion. Both these effects will have increased the coordination number of the atoms in the crystal lattice plane, which was accompanied by a decrease in the 
fringe spacing to $0.26 \mathrm{~nm}$, Figure 9 . The increase in diffraction intensity in the XRD data mentioned earlier could therefore be due to the formation of YAG particles with dominant (420) planes, which is the characteristic plane for single phase YAG.

However, it has been noted that once the (420) planes were formed, they will have created a lattice mismatch between the particles with (211) planes and hence retarded further particle growth. Wang et al. [29] stated that the crystals with different lattices tend to rotate to minimise the interface strain. This suggests that the particles with (420) planes could have rotated and minimised their interfacial strain rather than agglomerating when they contacted particles based on a (211) arrangement. This suggested that the particles with (420) planes were not involved in a self-assembled process to form a planar interface between the particles and therefore did not assist an Ostwald ripening process for particle growth.

Raising the synthesis conditions further to $300^{\circ} \mathrm{C}$ and $8.5 \mathrm{MPa}$ for $2 \mathrm{~h}$ led to only a slight increase, $\sim 4 \%$, in the diffraction intensity of the YAG powder, Figure $8 \mathrm{c}$. The STEM image displayed only particles with (420) planes and $0.26 \mathrm{~nm}$ fringe spacing, which suggests that the particles with (211) planes observed under the previous conditions were completely crystallised into single phase YAG particles. This implies that the structural rearrangement within the planes had reached a saturation limit and the particles with (420) planes were not self-assembling for facile coalescence. This, in turn, suggests that the surface energy of the (420) plane is not adequate to form necking between the adjacent particles under this condition; the arrows in Figure $8 \mathrm{c}$ indicate a clear particle-particle interface. 


\section{Conclusions}

When 1, 4 butanediol was used as the solvent medium for solvothermal synthesis at $300^{\circ} \mathrm{C}$ and $8.5 \mathrm{MPa}$ for $2 \mathrm{~h}$ with organic precursors, the XRD data showed that single phase YAG was obtained and TEM images revealed spherical and homogenous particle morphology, with a primary particle size of about $\sim 27 \mathrm{~nm}$. The BET measurements indicated a surface area of about $46.7 \pm 1 \mathrm{~m}^{2} \mathrm{~g}^{-1}$, which gave a calculated particle size that matched well with the TEM results. This suggested that there was little or no necking between adjacent particles and little agglomeration. The results also suggested that the particle growth kinetics occurred via a selfassembled Ostwald ripening process, with $7 \pm 1 \mathrm{~nm}$ aggregates formed at $270^{\circ} \mathrm{C}, 2$ MPa for $2 \mathrm{~h}$ coalescing into $\sim 27 \mathrm{~nm}$ particles by reducing their total interfacial energy along the $<112>$ crystallographic direction of the (211) planes. The coalesced particles will have undergone internal rearrangement of the atoms within the planes, transforming them into (420) planes. Once this has occurred, the (420) planes did not coalesce further since their surface energy was insufficient to form necks between the adjacent particles. Hence, the approach was successful in producing highly crystalline YAG powders of $\sim 27 \mathrm{~nm}$ in size with relatively little agglomeration compared to other synthesis routes described in the literature.

\section{Acknowledgement}

The authors would like to thank Morgan Advanced Materials, Swansea, UK, and Loughborough University Studentship for financial support. The authors also thank the LMCC staff at Loughborough University and colleagues in the Advanced Ceramics Group for their kind help and support during this project. 


\section{Reference List}

[1] T. Mah, Polycrystalline YAG; structural or functional? Journal of Ceramic Processing \& Research. 5 (2004) 369-379.

[2] L. Yang, M. Chen, Z. Lv, S. Wang, X. Liu, S. Liu, Preparation of a YAG: Ce phosphor glass by screen-printing technology and its application in LED packaging, Opt. Lett. 38 (2013) 2240-2243.

[3] S.W. Yoon, H.K. Park, J.H. Oh, Y.R. Do, Full Extraction of 2D Photonic Crystal Assisted: Ce Ceramic Plate Phosphor for Highly Efficient White LEDs, Photonics Journal, IEEE. 6 (2014) 1-10.

[4] G. Wei, Transparent ceramics for lighting, Journal of the European Ceramic Society. 29 (2009) 237-244.

[5] T.A. Parthasarathy, T. Mah, K. Keller, Creep mechanism of polycrystalline yttrium aluminum garnet, J Am Ceram Soc. 75 (1992) 1756-1759.

[6] P. Ramanujam, B. Vaidhyanathan, J. Binner, A. Anshuman, C. Spacie, A comparative study of the synthesis of nanocrystalline Yttrium Aluminium Garnet using sol-gel and co-precipitation methods, Ceram. Int. 40 (2014) 4179-4186.

[7] Q. Lu, W. Dong, H. Wang, X. Wang, A novel way to synthesize yttrium aluminum garnet from metal-inorganic precursors, J Am Ceram Soc. 85 (2002) 490-492.

[8] J. Li, T. Ikegami, J. Lee, T. Mori, Y. Yajima, Co-precipitation synthesis and sintering of yttrium aluminum garnet (YAG) powders: the effect of precipitant, Journal of the European Ceramic Society. 20 (2000) 2395-2405.

[9] P.S. Devi, Y. Lee, J. Margolis, J.B. Parise, S. Sampath, H. Herman, J.C. Hanson, Comparison of citrate-nitrate gel combustion and precursor plasma spray processes for the synthesis of yttrium aluminum garnet, J. Mater. Res. 17 (2002) 2846-2851.

[10] J. Li, Y. Pan, F. Qiu, Y. Wu, J. Guo, Nanostructured Nd: YAG powders via gel combustion: the influence of citrate-to-nitrate ratio, Ceram. Int. 34 (2008) 141-149.

[11] D. Segal, Chemical synthesis of ceramic materials, J.Mater.Chem. 7 (1997) 1297-1305.

[12] A. Cabanas, J. Li, P. Blood, T. Chudoba, W. Lojkowski, M. Poliakoff, E. Lester, Synthesis of nanoparticulate yttrium aluminum garnet in supercritical water-ethanol mixtures, The Journal of supercritical fluids. 40 (2007) 284-292.

[13] X. Zhang, H. Liu, W. He, J. Wang, X. Li, R.I. Boughton, Synthesis of monodisperse and spherical YAG nanopowder by a mixed solvothermal method, $\mathrm{J}$. Alloys Compounds. 372 (2004) 300-303.

[14] M. Inoue, H. Otsu, H. Kominami, T. Inui, Synthesis of yttrium aluminum garnet by the glycothermal method, J Am Ceram Soc. 74 (1991) 1452-1454. 
[15] R. Kasuya, T. Isobe, H. Kuma, J. Katano, Photoluminescence enhancement of peg-modified YAG: Ce3 nanocrystal phosphor prepared by glycothermal method, The Journal of Physical Chemistry B. 109 (2005) 22126-22130.

[16] M. Nyman, L.E. Shea-Rohwer, J.E. Martin, P. Provencio, Nano-YAG: Ce mechanisms of growth and epoxy-encapsulation, Chemistry of Materials. 21 (2009) 1536-1542.

[17] R. Kasuya, T. Isobe, H. Kuma, Glycothermal synthesis and photoluminescence of YAG: Ce 3 nanophosphors, J. Alloys Compounds. 408 (2006) 820-823.

[18] J. Li, T. Ikegami, Y. Wang, T. Mori, 10-mol\%-Gd2O3-Doped CeO2 Solid Solutions via Carbonate Coprecipitation: A Comparative Study, J Am Ceram Soc. 86 (2003) 915-921.

[19] S. Stein, NIST Standard Reference Database 1a. (2005).

[20] X. Yang, P. Neta, S.E. Stein, Quality control for building libraries from electrospray ionization tandem mass spectra, Anal. Chem. 86 (2014) 6393-6400.

[21] L.S. Al Hashmi, M.A. Hossain, A.M. Weli, Q. Al-Riyami, J.N. AlSabahi, Gas chromatography-mass spectrometry analysis of different organic crude extracts from the local medicinal plant of Thymus vulgaris L, Asian Pacific journal of tropical biomedicine. 3 (2013) 69-73.

[22] L.C. Wilson, H.L. Wilson, W.V. Wilding, G.M. Wilson, Critical point measurements for fourteen compounds by a static method and a flow method, Journal of Chemical \& Engineering Data. 41 (1996) 1252-1254.

[23] H. Borchert, E.V. Shevchenko, A. Robert, I. Mekis, A. Kornowski, G. Grübel, H. Weller, Determination of nanocrystal sizes: a comparison of TEM, SAXS, and XRD studies of highly monodisperse CoPt3 particles, Langmuir. 21 (2005) 1931-1936.

[24] S.E. Hunter, C.E. Ehrenberger, P.E. Savage, Kinetics and mechanism of tetrahydrofuran synthesis via 1, 4-butanediol dehydration in high-temperature water, J. Org. Chem. 71 (2006) 6229-6239.

[25] W. Li, E. Ding, Preparation and characterization of a novel solid-liquid PCM: Butanediol di-stearate, Mater Lett. 61 (2007) 1526-1528.

[26] M.W. Peters, J.D. Taylor, D.E. Henton, L.E. Manzer, P.R. Gruber, J.M. Griffith, Y. Al Obaidi, Integrated methods of preparing renewable chemicals. (2013).

[27] Y. Liu, A.M. Malek, D. Coffey, E.E. Stangland, A.E. Schweizer, Catalytic composition for production of olefins with decreased oxygenate byproducts. (2011).

[28] H. Wilmer, M. Kurtz, K. Klementiev, O. Tkachenko, W. Grünert, O. Hinrichsen, A. Birkner, S. Rabe, K. Merz, M. Driess, Methanol synthesis over ZnO: A structuresensitive reaction? Physical Chemistry Chemical Physics. 5 (2003) 4736-4742. 
[29] Z.L. Wang, X. Feng, Polyhedral shapes of CeO2 nanoparticles, The Journal of Physical Chemistry B. 107 (2003) 13563-13566.

[30] A. Igarashi, N. Ichikawa, S. Sato, R. Takahashi, T. Sodesawa, Dehydration of butanediols over $\mathrm{CeO} 2$ catalysts with different particle sizes, Applied Catalysis A: General. 300 (2006) 50-57.

[31] T. Mishima, R. Tanaka, S. Kohsaka, K. Koga, Grain Boundary Analysis of Silicon Nitride Materials, in: Anonymous Grain Boundary Controlled Properties of Fine Ceramics, Springer, 1992, pp. 62-69.

[32] S.B. Thoma, D.M. Smith, J. Boughton, R. Davies, On-line Surface Area Measurement of Concentrated Slurries using low field spin-lattice relaxation NMR, Particle \& particle systems characterization. 10 (1993) 246-251.

[33] A.J. Houtepen, R. Koole, D. Vanmaekelbergh, J. Meeldijk, S.G. Hickey, The hidden role of acetate in the PbSe nanocrystal synthesis, J. Am. Chem. Soc. 128 (2006) 6792-6793.

[34] F. Bamiduro, M.B. Ward, R. Brydson, S.J. Milne, Hierarchical Growth of ZnO Particles by a Hydrothermal Route, J Am Ceram Soc. 97 (2014) 1619-1624.

[35] J.H. Yu, J. Joo, H.M. Park, S. Baik, Y.W. Kim, S.C. Kim, T. Hyeon, Synthesis of quantum-sized cubic ZnS nanorods by the oriented attachment mechanism, J. Am. Chem. Soc. 127 (2005) 5662-5670.

[36] H. Roh, D.H. Kim, I. Park, H.J. Song, S. Hur, D. Kim, K.S. Hong, Template-free synthesis of monodispersed Y 3 Al 5 O 12: Ce 3 nanosphere phosphor, Journal of Materials Chemistry. 22 (2012) 12275-12280.

[37] J. Qin, R. Yang, G. Liu, M. Li, Y. Shi, Grain growth and microstructural evolution of yttrium aluminum garnet nanocrystallites during calcination process, Mater. Res. Bull. 45 (2010) 1426-1432.

[38] U. Aschauer, P. Bowen, S.C. Parker, Surface and mirror twin grain boundary segregation in Nd: YAG: an atomistic simulation study, J Am Ceram Soc. 91 (2008) 2698-2705.

[39] W.M. Haynes, CRC Handbook of Chemistry and Physics, CRC press, 2014.

[40] K. Bhattacharya, S. Conti, G. Zanzotto, J. Zimmer, Crystal symmetry and the reversibility of martensitic transformations, Nature. 428 (2004) 55-59.

[41] R. Aso, D. Kan, Y. Shimakawa, H. Kurata, Atomic level observation of octahedral distortions at the perovskite oxide heterointerface, Scientific reports. 3 (2013). 